\title{
A general relativistic model of galaxy bulges in GEDi paradigm
}

\author{
C. H. Coimbra-Araújo ${ }^{1}$ and P. S. Letelier ${ }^{2}$ \\ ${ }^{1}$ Instituto de Física "Gleb Wataghin", Universidade Estadual de Campinas, Unicamp, \\ 13083-970, Campinas, SP, Brazil. \\ ${ }^{2}$ Departamento de Matemática Aplicada, Instituto de Matemática, Estatística e Computação \\ Científica, Universidade Estadual de Campinas, Unicamp, 13083-970, Campinas, SP, Brazil.
}

\begin{abstract}
Recently a new approach was presented where astronomical objects as galaxies and clusters are idealized as self-gravitating systems living in a universe endowed with more than 3+1 dimensions. A such paradigm, named GEDi (Gravitation with Extra Dimensions), may solve exactly the missing mass problem for rotation curves of galaxies or gravitational lensing of clusters with no dark matter particles. Here we present an introductory discussion about the construction of a real galaxy using a Miyamoto-Nagai solution for isotropical coordinates to mimic spiral galaxies with bulges.
\end{abstract}

Keywords. galaxies: general, dark matter, galaxies: bulges, extra dimensions

\section{Introduction}

Recently, a general model of Gravitation with Extra Dimensions (GEDi, CoimbraAraújo \& Letelier 2006; Coimbra-Araújo \& Letelier 2007a, 2007b), was derived from the construction of self-gravitating objects living in a space-time endowed with extra dimensions. Actually, the effective modification of the gravity law induced by GEDi could explain the observed flatness of galaxy rotation curves equally well as the usually given dark matter interpretation. The inclusion of extra dimensions also leads to better fits of the gravitational lensing data for galaxy clusters, without changing results obtained in solar system scales. A novel physical interpretation arises from the geometrical issues of a such model, that are distinct of braneworld approaches, and indeed astrophysical objects appear to be sensitive to extra dimensions according to a $\mathrm{R} / \mathrm{M}$ (length per mass) scale law.

\section{Thin disks of galaxies in GEDi}

Rotation curves of thin disks, surface densities and pressures were derived from the multidimensional solution for a Weyl metric

$$
\mathrm{d} s^{2}=\sum_{i=1}^{D-2} \epsilon_{i} e^{2 \Phi_{i}}\left(\mathrm{~d} x^{i}\right)^{2}+g_{a b} \mathrm{~d} y^{a} \mathrm{~d} y^{b},
$$

combined with a distributional technique (Coimbra-Araújo \& Letelier 2007a). The main results for the circular geodesics on the plane of the disk, after an accurate study of stability, is showed in the Fig. 1. 


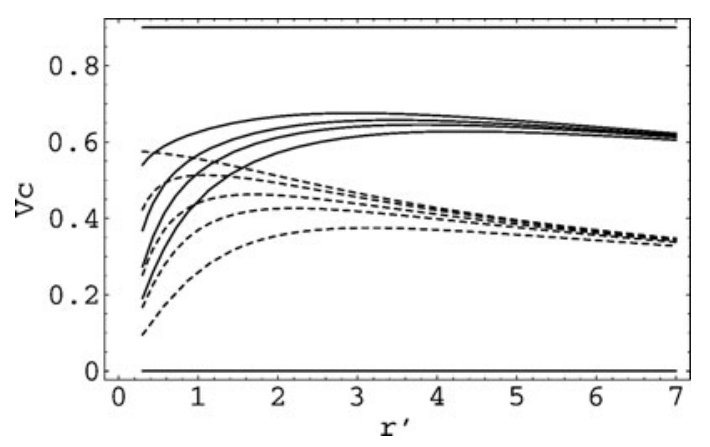

Figure 1. Stable rotation curves for thin disks. The dotted ones are the Newtonian-like (where there are no extra dimensions), and the full ones are the stable curves derived admitting two extra dimensions. It is awaited that the same occurs for systems that mimics galaxies with bulges.

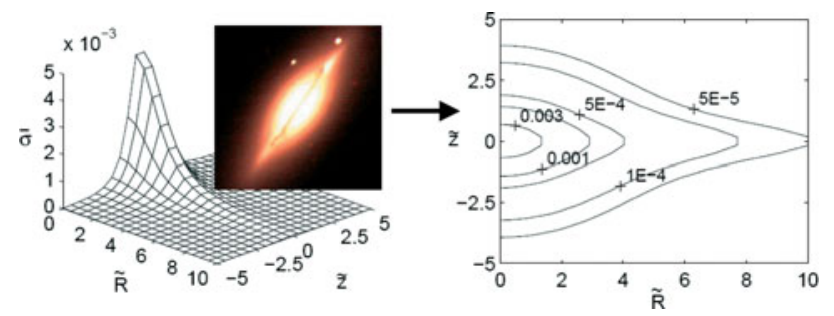

Figure 2. The density profiles for a isotropic configuration by Miyamoto-Nagai solutions. A such system could mimic a real spiral galaxy with a central bulge.

\section{Miyamoto-Nagai systems and concluding remarks}

To construct a model of galaxy including bulges, the next step is to superpose some spherical solution to the thin disk potential or work with a most effective coordinate system (see e.g. Vogt \& Letelier 2003; Vogt \& Letelier 2005). An excellent choice is the isotropical coordinate system. Density profiles of a such configuration, solved in a Miyamoto-Nagai disk approach can be extended to a case of a multidimensional configuration (Fig. 2).

In particular, the solvability for rotation curves in the GEDi framework is sensitive to $\mathrm{R} / \mathrm{M}$ (length per mass) scales. The nature of such results must be discussed in forthcoming articles with more detail and a preliminary interpretation points that the above model could be an alternative to dark matter (substituting the concept of missing mass).

\section{Acknowledgements}

We would like to acknowledge the financial support of IAU and CAPES.

\section{References}

Coimbra-Araújo, C. H. \& Letelier, P. S. 2006, in: V. Karas (ed.), Proceedings of IAU Symposium 238, General relativistic results for a galactic disc in a multidimensional space-time (Cambridge: Cambridge Un. Press), vol. 238, p. 343

Coimbra-Araújo, C. H. \& Letelier, P. S. 2007a, Phys. Rev. D 76, 043522

Coimbra-Araújo, C. H. \& Letelier, P. S. 2007b, Class. Quantum Grav. (in press)

Vogt, D. \& Letelier, P. S. 2003, Phys. Rev. D 68, 084010

Vogt, D. \& Letelier, P. S. 2005, MNRAS 363, 268 